01

\title{
Квантово-размерная зависимость энергии образования вакансии в заряженных малых металлических кластерах. Капельная модель
}

\author{
(C) В.В. Погосов, В.И. Рева
}

Запорожский национальный технический университет, Запорожье, Украина

E-mail: vpogosov@zntu.edu.ua

(Поступила в Редакцию 7 ноября 2017 г.)

\begin{abstract}
Выполнены самосогласованные вычисления энергии образования моновакансии для сферических кластеров $\mathrm{Na}_{N}, \mathrm{Mg}_{N}, \mathrm{Al}_{N}(12<N \leq 168)$ в капельной модели стабильного желе. Рассмотрены сценарии образования вакансии по Шоттки и „выдуванию пузырька-вакансии“. Показано, что асимптотическое поведение размерных зависимостей энергии образования вакансии по этим двум механизмам отличаются друг от друга, а разница между характеристиками заряженного и нейтрального кластера целиком определяется разницей потенциалов ионизации кластеров и энергий прилипания к ним электронов.
\end{abstract}

DOI: $10.21883 /$ FTT.2018.04.45668.315

1. В условиях термодинамического равновесия концентрация вакансий в металлах вычисляется при наличии энергии образования вакансии, которую либо извлекают из спектра аннигиляции свободных и локализованных в вакансиях позитронов, либо рассчитывают в различных моделях $[1,2]$. В точке плавления относительная концентрация равновесных вакансий в металлах составляет доли процента, однако в случае радиационных повреждений относительная концентрация неравновесных вакансий может достигать тридцати процентов. Молекулярно-динамическое моделирование демонстрирует понижение температуры плавления твердых тел с вакансиями по сравнению с плавлением в идеальном образце [3].

В экспериментах показано, что температура плавления малых металлических кластеров характеризуется сильной осцилляционной размерной зависимостью, а диффузия поверхностных вакансий в объем (во время предплавления) более выгодна для кластеров с незаполненными электронными оболочками, чем для кластеров с магическим числом атомов [4]. Эти факты стимулируют повышенный интерес к описанию фазового перехода из твердого в жидкое состояние в качестве конфигурационного возбуждения вакансий в кластерах.

Перспективным объектом наноэлектроники являются металлические гранулы (островки, кластеры атомов), связанные слабыми туннельными взаимодействиями $[5,6]$.

Известно, что сверхпроводящие корреляции электронов зависят от плотности состояний вблизи энергии Ферми. В малых металлических кластерах с замкнутыми электронными оболочками (магические кластеры или суператомы) имеется сильное вырождение уровней и высокая плотность состояний, что благоприятствует переходу в сверхпроводящее состояние [7]. Исследования кластеров $\mathrm{Al}_{32-95}$ [8] показали, что в $\mathrm{Al}_{37,44,66,68}$ уже при температуре в $100 \mathrm{~K}$ начинаются процессы фор- мирования устойчивых куперовских пар. В перспективе возможно создание высокотемпературных сверхпроводящих туннельных кластерных цепочек и, соответственно, макроскопической сверхпроводимости при высокой температуре. Кластеры, содержащие вакансии, могут иметь, как будет показано ниже, иные магические числа атомов, чем бездефектные. Это может приводить к размытию влияния оболочечных эффектов на сверхпроводящие корреляции, включая щель спектра возбуждения.

Для вычисления энергии образования вакансии в кластерах и влияние на нее квантования электронного спектра мы использовали модель стабильного желе [9], которая не содержит подгоночных параметров.

Размерные зависимости для энергий образования моновакансии рассчитывались по двум сценариям: Шоттки и „выдувания пузырька“ [10]. Показано, что асимптотическое поведение размерных зависимостей для этих механизмов отличаются друг от друга и слабо зависят от числа атомов в кластере, а разница между характеристиками заряженного и нейтрального кластера полностью определяется размерными зависимостями потенциала ионизации IP кластера и энергии прилипания электронов ЕА. Для небольших кластеров $\mathrm{Rb}, \mathrm{K}, \mathrm{Na}$, $\mathrm{Li}, \mathrm{Mg}$ и $\mathrm{Al}$ с $N \leq 270$, содержащих моновакансию, методом Кона-Шема рассчитаны профили распределений плотности электронов и эффективного потенциала; потенциала ионизации и энергии прилипания электронов; энергии образования вакансии. В данной работе для демонстрации и анализа результатов мы ограничились кластерами металлов $\mathrm{Na}, \mathrm{Mg}$ и $\mathrm{Al} \mathrm{c} N \leq 168$.

2. В соответствии со сценарием Шоттки из сплошной $N$-атомной сферы радиуса $R_{N}=N^{1 / 3} r_{0}$ и полной энергией $E_{N}$ удаляется на бесконечность один атом; $r_{0}-$ радиус ячейки Вигнера-Зейтца. В конечном состоянии имеем сферу такого же радиуса $\left(R_{N-1, v}=R_{N}\right)$, но с энергией $E_{N-1}, v$, содержащей $N-1$ атомов и одну вакансию радиусом $r_{0}$ в центре сферы. Шаровой слой 
между $r=r_{0}$ и $r=R_{N-1, v}$ содержит $N-1$ атомов. Тогда для заряженной зарядом $Q$ сферы энергия образования вакансии определяется выражением

$$
\varepsilon_{N, v}^{\mathrm{vac}, \mathrm{Sh}, Q}=\left[E_{N-1, v}^{Q}+E_{\mathrm{at}}\right]-E_{N}^{Q},
$$

где $E_{\text {at }}$ - энергия атома (желе-сферы радиуса $r_{0}$ ) в вакууме. Самосогласованные значения $E_{\mathrm{at}}=-5.0961$, -23.6061 и $-53.2841 \mathrm{eV}$ для $\mathrm{Na}, \mathrm{Mg}$ и $\mathrm{Al}$ соответственно.

Суть другого механизма, при котором число атомов в сфере не меняется [10], в „выдувании вакансии“ (сферической дырки радиуса $r_{0}$ в центре сферы):

$$
\varepsilon_{N, v}^{\text {vac,blow, } Q}=E_{N, v}^{Q}-E_{N}^{Q} .
$$

Исследуем асимптотику энергии образования вакансии. Для этого воспользуемся выражениями для энергии когезии атома и энергии образования вакансии для $3 \mathrm{D}$ металла $[9,10]$ в капельной модели (LDM) и приближении локальной плотности (LDA)

$$
\begin{aligned}
& \varepsilon^{\mathrm{coh}}\left(r_{0}\right)=4 \pi r_{0}^{2} \sigma_{0}\left(1+\tilde{\delta}_{1}+\tilde{\delta}_{2}\right) \equiv \varepsilon_{\infty}^{\mathrm{coh}}, \\
& \varepsilon^{\mathrm{vac}}\left(r_{0}\right)=4 \pi r_{0}^{2} \sigma_{0}\left(1-\tilde{\delta}_{1}+\tilde{\delta}_{2}\right) \equiv \varepsilon_{\infty}^{\mathrm{vac}},
\end{aligned}
$$

где $\sigma_{0}-$ удельная поверхностная энергия поверхности нулевой кривизны, а также первая $\tilde{\delta}_{1} \equiv \delta_{1} / r_{0}$ и вторая $\tilde{\delta}_{2} \equiv \delta_{2} / r_{0}^{2}$ размерные поправки к ней; $r_{0}=3.99,3.34$ и $2.99 a_{0}$ для $\mathrm{Na}, \mathrm{Mg}$ и $\mathrm{Al}$ соответственно, $a_{0}$ - боровский радиус.

Величины $\varepsilon_{\infty}^{\mathrm{vac}}$ вычислены нами в [11] по теории рассеяния электронных волн на самосогласованном электронвакансионном потенциале, $\varepsilon_{\infty}^{\mathrm{vac}}=0.33,0.72$ и $1.00 \mathrm{eV}$ для $\mathrm{Na}, \mathrm{Mg}$ и $\mathrm{Al}$ соответственно. Используя их, а также величины $\varepsilon_{\infty}^{\mathrm{coh}}, \tilde{\delta}_{2}=-0.13(\mathrm{Na}),-0.015(\mathrm{Mg})$ и $+0.22(\mathrm{Al})$ из [9], находим, что $\tilde{\delta}_{1}=0.32(\mathrm{Na}), 0.54(\mathrm{Mg})$ и $0.57(\mathrm{Al})$. Величины $\delta_{1}$ и $\delta_{2}$ нам необходимы для построения асимптотики энергии образования вакансии. Она определяется разностью полных энергий сфер, рассчитанных по формулам (1) и (2) в пределе больших $N$, и сводится к разности полных поверхностных энергий этих сфер. Вследствие того, что $Q= \pm e$, влияние зарядки кластеров на асимптотики ничтожно $(-e-$ заряд электрона).

Для механизма выдувания вакансии получим

$$
\begin{aligned}
\varepsilon_{N, v}^{\mathrm{vac}, \text { blow }}= & 4 \pi R_{N, v}^{2} \sigma_{0}\left(1+\frac{\delta_{1}}{R_{N, v}}+\frac{\delta_{2}}{R_{N, v}^{2}}\right) \\
& +\varepsilon^{\mathrm{vac}}\left(r_{0}\right)-4 \pi R_{N}^{2} \sigma_{0}\left(1+\frac{\delta_{1}}{R_{N}}+\frac{\delta_{2}}{R_{N}^{2}}\right) \\
= & \varepsilon_{\infty}^{\mathrm{vac}}\left(1+\frac{2}{3\left(1-\tilde{\delta}_{1}+\tilde{\delta}_{2}\right) N^{1 / 3}}\right) .
\end{aligned}
$$

Асимптотика (4) демонстрирует уменьшение энергии образования вакансии с ростом $N$, что согласуется с выводами работ $[12,13]$, но противоречит результатам [14-16]. Представляя выражение (4) в виде

$$
\varepsilon_{N, v}^{\text {vac,blow }}=\varepsilon_{\infty}^{\text {vac }}\left(1+\frac{A}{N^{1 / 3}}\right),
$$

имеем $A=1.21,1.50$ и 1.03 для $\mathrm{Na}, \mathrm{Mg}$ and $\mathrm{Al}$ соответственно. Это качественно согласуется со значениями $A=1.33$ и 1.46 для Сu и $\beta-$ Ті из [12].

Для механизма Шоттки асимптотика $\varepsilon_{N, v}^{\mathrm{vac}, \mathrm{Sh}}$ определяется суммой выражения (4) и асимптотики энергии когезии

$$
\varepsilon_{N}^{\mathrm{coh}}=\varepsilon_{\infty}^{\mathrm{coh}}-\frac{2 \sigma_{0}}{n_{\mathrm{at}} R_{N}}
$$

где $n_{\mathrm{at}}=3 /\left(4 \pi r_{0}^{3}\right)$ - концентрация атомов в 3D металле.

По определению, потенциал ионизации IP и энергия прилипания электронов ЕА выражается в виде разности полных энергий:

$$
\begin{gathered}
\mathrm{IP}_{N, v}=E_{N, v}^{+e}-E_{N, v}^{0}, \\
\mathrm{EA}_{N, v}=E_{N, v}^{0}-E_{N, v}^{-e} .
\end{gathered}
$$

Используя определения (1), (2) и (6), разницу между энергиями образования вакансии в заряженном и нейтральном кластере можно записать как

$$
\begin{gathered}
\Delta \varepsilon_{N, v}^{\mathrm{vac}, \mathrm{Sh},+e}=\mathrm{IP}_{N-1, v}-\mathrm{IP}_{N}, \\
\Delta \varepsilon_{N, v}^{\mathrm{vac}, \mathrm{Sh},-e}=\mathrm{EA}_{N}-\mathrm{EA}_{N-1, v}, \\
\Delta \varepsilon_{N, v}^{\mathrm{vac}, \text { blow },+e}=\mathrm{IP}_{N, v}-\mathrm{IP}_{N}, \\
\Delta \varepsilon_{N, v}^{\text {vac,blow },-e}=\mathrm{EA}_{N}-\mathrm{EA}_{N, v} .
\end{gathered}
$$

В соответствии с теоремой Купменса, формулы (6) можно переписать в виде

$$
\begin{gathered}
\mathrm{IP}_{N, v}=-\varepsilon_{N, v}^{\mathrm{HO}}+\frac{\left(+e^{2}\right)}{2 C_{N, v}^{+}}, \\
\mathrm{EA}_{N, v}=-\varepsilon_{N, v}^{\mathrm{LU}}-\frac{\left(-e^{2}\right)}{2 C_{N, v}^{-}},
\end{gathered}
$$

где $\varepsilon_{N, v}^{\mathrm{HO}} / \varepsilon_{N, v}^{\mathrm{LU}}$ и $C_{N, v}^{+}-$энергии верхних занятых/нижних незанятых электронных орбиталей кластера и электрические емкости соответственно. Выражения (8) удобны для анализа прямых вычислений по (6) и (7).

Формулы для $\mathrm{IP}_{N}$ и $\mathrm{EA}_{N}$ бездефектных кластеров получаются из (6) и (8) заменой нижних индексов $N, v \rightarrow N$.

3. На рис. 1 приведены результаты вычислений размерных зависимостей энергии образования вакансий по двум механизмам, сравнение которых, в частности, подтверждает энергетическую выгодность механизма выдувания вакансии. Для данного интервала $N$ эти зависимости сильно осциллируют, что является результатом квантования спектра электронов и заполнения электронных оболочек. Неожиданным оказалось то, что в асимптотике размерная зависимость $\varepsilon_{N, v}^{\mathrm{vac}, \mathrm{Sh}}$ фактически отсутствует, т. е. зависимость (4) подавляется зависимостью (5). 

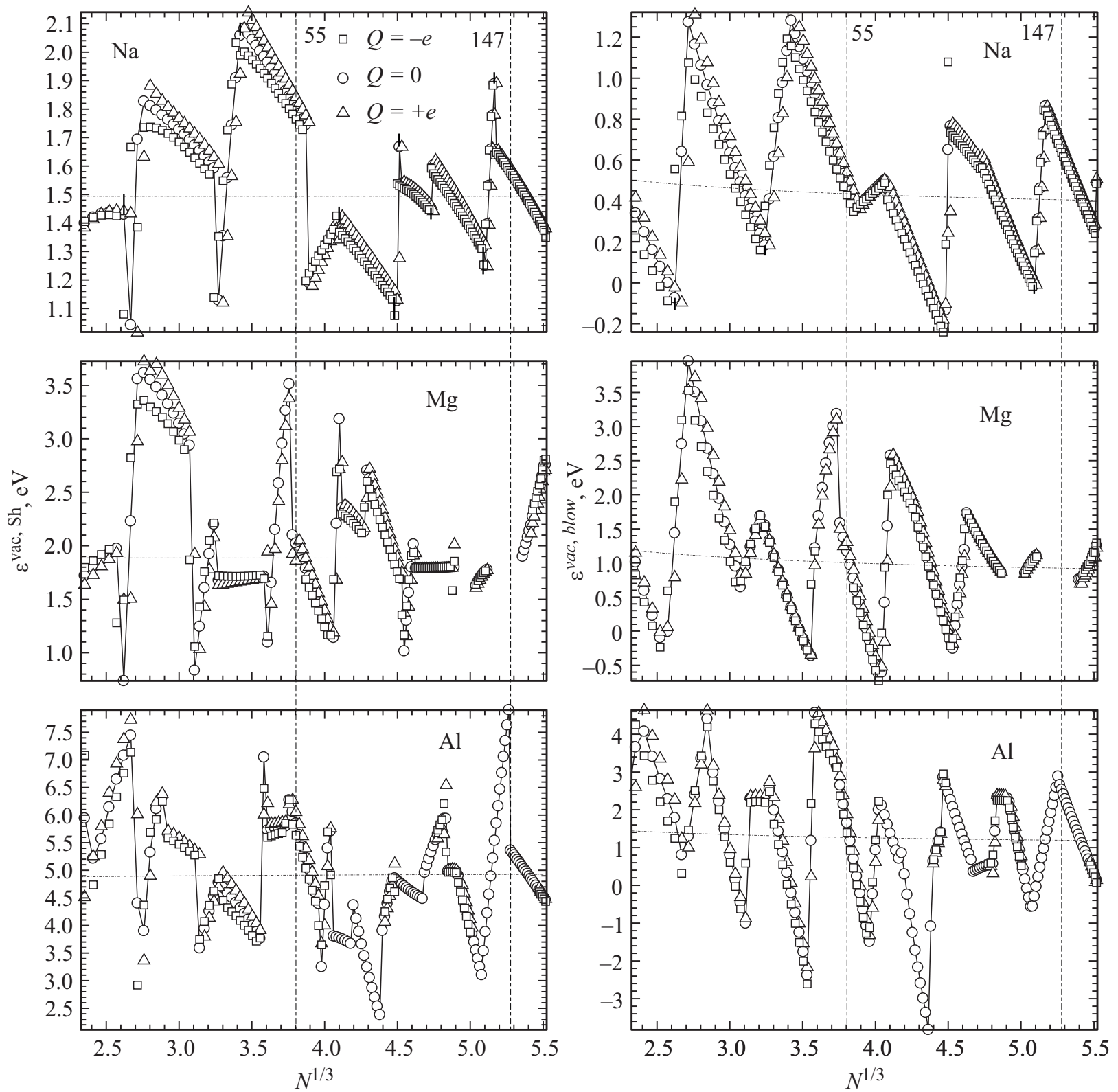

Рис. 1. Рассчитанные энергии образования моновакансии $\varepsilon_{N, v}^{\mathrm{vac}, \text { Sh }}(1)$ и $\varepsilon_{N, v}^{\mathrm{vac}, \text { blow }}(2)$ в заряженных и нейтральных кластерах; штрихпунктирные линии - асимптотики.

В работе [17] сообщается о результатах $a b$ initio вычислений для нейтральных $(Q=0)$ кластеров

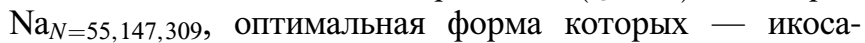
эдр. Числа $N=55,147$ и 309 соответствуют 2, 3 и 4 атомным оболочкам-слоям бездефектных кластеров. Показано также, что энергия образования моновакансии в кластере существенно зависит от места образования вакансии, а также от финальной позиции перемещенного атома. В работе [17] по одному из сценариев удаление атома происходит на бесконечность, в вакуум, а по другому - на плоский участок поверхности, ребро или вершину икосаэдра. Первый сценарий работы [17] совпадает со сценарием Шоттки, использованным в нашей работе, а второй можно лишь приблизительно сопоставить с механизмом „выдувания пузырька“, поскольку в последнем все атомы кластера сдвигаются со своих мест, коллективно создавая вакансию в центре кластера.

В табл. 1 для сравнения приведены энергии образования вакансий. Несмотря на то, что сравнение значений

Таблица 1. Энергии образования вакансий $(\mathrm{eV})$ в нейтральных кластерах $\mathrm{Na}_{N}$

\begin{tabular}{r|c|c|c|c}
\hline \multicolumn{1}{c|}{$N$} & \multicolumn{2}{|c|}{$\varepsilon_{N, v}^{\mathrm{vac}, \mathrm{Sh}}$} & \multicolumn{2}{|c}{$\varepsilon_{N, v}^{\text {vac,blow }}$} \\
\hline 55 & 1.80 & $1.35^{a}$ & 0.49 & $0.49^{a}$ \\
147 & 1.58 & $1.18^{a}$ & 0.66 & $0.43 / 0.63^{a}$
\end{tabular}

Примечание. ${ }^{a}$ ab initio вычисления [17]. 
Таблица 2. Расчетные значения (в eV) потенциала ионизации энергии прилипания и верхних/нижних занятых/незанятых уровней, а также разницы энергий образования вакансий заряженных и нейтральных кластеров $\mathrm{Na}_{32-36} \mathrm{~L}_{60-64}$

\begin{tabular}{l|c|c|c|c|c|c|c|c|c|c}
\hline$N$ & $\mathrm{IP}_{N, v}$ & $-\varepsilon_{N, v}^{\mathrm{HO}}$ & $\mathrm{EA}_{N, v}$ & $-\varepsilon_{N, v}^{\mathrm{LU}}$ & $\mathrm{IP}_{N}$ & $-\varepsilon_{N}^{\mathrm{HO}}$ & $\mathrm{EA}_{N}$ & $-\varepsilon_{N, v}^{\mathrm{LU}}$ & $\Delta \varepsilon_{N}^{\mathrm{vac}, \text { blow, }+e}$ & $\Delta \varepsilon_{N}^{\mathrm{vac}, \text { blow, }-e}$ \\
\hline 32 & 3.977 & 3.043 & 2.079 & 3.043 & 3.948 & 2.964 & 1.994 & 2.964 & 0.029 & -0.085 \\
33 & 4.041 & 3.075 & 2.118 & 3.075 & 3.976 & 3.000 & 2.038 & 3.000 & 0.065 & -0.080 \\
$34^{*}$ & 4.063 & 3.104 & 1.471 & 2.386 & 4.002 & 3.034 & 1.664 & 2.609 & 0.061 & 0.193 \\
35 & 3.360 & 2.435 & 1.529 & 2.435 & 3.584 & 2.630 & 1.695 & 2.630 & -0.224 & 0.166 \\
36 & 3.396 & 2.480 & 1.582 & 2.480 & 3.592 & 2.649 & 1.723 & 2.649 & -0.196 & 0.141 \\
\hline 60 & 5.782 & 4.699 & 3.618 & 4.699 & 5.586 & 4.497 & 3.409 & 4.497 & 0.196 & -0.209 \\
61 & 5.813 & 4.734 & 3.657 & 4.734 & 5.624 & 4.539 & 3.456 & 4.539 & 0.189 & -0.201 \\
$62^{*}$ & 5.843 & 4.769 & 2.893 & 3.936 & 5.662 & 4.582 & 3.26 & 4.325 & 0.181 & 0.367 \\
63 & 5.053 & 4.013 & 2.979 & 4.013 & 5.408 & 4.348 & 3.293 & 4.348 & -0.355 & 0.314 \\
64 & 5.116 & 4.084 & 3.059 & 4.084 & 5.422 & 4.37 & 3.325 & 4.370 & 0.306 & 0.266
\end{tabular}

Приме чание. Звездочкой обозначены магические числа атомов $N^{*}$.

$\varepsilon_{N, v}^{\mathrm{vac}, \mathrm{Sh}}$ показывает разницу примерно в $0.4-0.5 \mathrm{eV}$, размерная зависимость ab initio вычислений [17] воспроизводится в нашей простой модели.

Значения $\varepsilon_{N, v}^{\mathrm{vac}, \text { blow }}$ согласуются лучше. Чтобы продемонстрировать зависимость $\varepsilon_{N, v}^{\text {vac,blow }}$ от местоположения вакансии для $N=147$, приведены два значения: 0.43/0.63 eV, которые соответствуют перемещению атома из центра/из первой атомной оболочки икосаэдра на плоский участок его поверхности.

На рис. 2 приведены рассчитанные по (6) в LDM и LDA зависимости $\operatorname{IP}(N)$ и $\operatorname{EA}(N)$. Значения $\varepsilon_{N, v}^{\text {vac,blow }}$ на рис. 1 становятся отрицательными для кластеров с такими $N$, при которых максимальный вклад дают уровни с низкими орбитальными числами $l(s, p-$ и частично $d$-орбитали). На рис. 2 эти узкие области заключены между вертикальными штриховыми линиями.

Магические числа $N^{*}$ атомов для идеальных кластеров и кластеров с моновакансией различны: $N^{*}=1,4$, $9,10,17,20,29,34,45,46,53,66,69,78,93,98$, (99), $\{115\},(116),\{126\}, 127,134$ для $\mathrm{Mg}$ и $N^{*}=6$, (30), 44, 46, 52, 62, \{66\}, (84), (102), \{104\}, \{136\}, 146, $\{154\}$ для Al. В круглых скобках приведены числа для дефектных кластеров, которые не совпадают с числами $N^{*}$ для бездефектных кластеров, а в фигурных скобках наоборот. Для $\mathrm{Na} N^{*}=2,8,18,20,34,40,58,68,90,92$, 106, 132, 138, 168.

С помощью рис. 2, на котором приведены зависимости $\operatorname{IP}(N)$ и $\operatorname{EA}(N)$ для кластеров $\mathrm{Na}, \mathrm{Mg}$ и $\mathrm{Al}$, несложно проверить, что разности энергий в (7) полностью определяются поведением $\mathrm{IP}(N)$ и $\mathrm{EA}(N)$.

Для иллюстрации в табл. 2 приведены значения IP и ЕА, рассчитанные непосредственно по (6) для некоторых выделенных кластеров. Также приведены значения $\varepsilon^{\mathrm{HO}}$ и $\varepsilon^{\mathrm{LU}}$, фигурирующие в (8) и вычисленные в результате совместного решения системы уравнений Кона-Шема и уравнения Пуассона. Сравнением IP, EA и $\varepsilon^{\mathrm{HO}}, \varepsilon^{\mathrm{LU}}$ можно установить величину энергии зарядки и емкости кластеров. Как оказалось, разницы энергий образования вакансий в заряженных и нейтральных кластерах почти целиком определяются разностями $\varepsilon^{\mathrm{HO}}$,
$\varepsilon^{\mathrm{LU}}$, а при переходе через $N=N^{*}$ эти разности меняют знак, т. е. на рис. 1 квадраты и треугольники относительно кружков меняются местами по вертикали.

4. В данной работе рассчитаны квантово-размерные зависимости энергии образования внутренней вакансии по механизмам Шоттки и „выдуванию пузырька“. Размерные асимптотики этих двух механизмов отличны друг от друга и слабо зависят от числа атомов в кластере. Квантово-размерные зависимости энергии образования вакансии для малых кластеров содержат локальные максимумы. Магические кластеры, соответствующие им, являются более устойчивыми, т.е. имеют бо́льшие по величине энергии связи и образования вакансии, чем их соседи. Такими максимумами с ростом $N$ завершается заполнение очередной электронной оболочки. С ростом $N$, на участках между максимумами, энергия образования вакансий монотонно убывает.

Разница между характеристиками заряженного и нейтрального кластера точно определяется разностью потенциалов ионизации и энергий прилипания электронов. Магические числа атомов для идеальных кластеров $(\mathrm{Mg}, \mathrm{Al})$ и кластеров с моновакансией различны, особенно для $\mathrm{Al}$, но не для $\mathrm{Na}$.

Анализ экспериментов по плавлению кластеров и соответствующих вычислений [18-20] указывает на сложную картину взаимосвязей между геометрическими и электронными оболочечными эффектами и свидетельствует о том, что квантово-механическое описание является решающим для количественного понимания изменений в температуре плавления, наблюдаемых для свободных кластеров. Вследствие того, что характеристики заряженных и нейтральные кластеров отличаются друг от друга, разумно предположить, что с помощью вариации избыточного заряда можно управлять температурой плавления кластеров.

Энергия образования внутренней вакансии зависит от ее местоположения и сценария ее создания [17]. Еще в большей мере это относится к поверхностным вакансиям, а поэтому в аналитическом виде невозможно оценить влияние всех вакансий на энергетику кластера, тем более, что концентрация их неизвестна. 


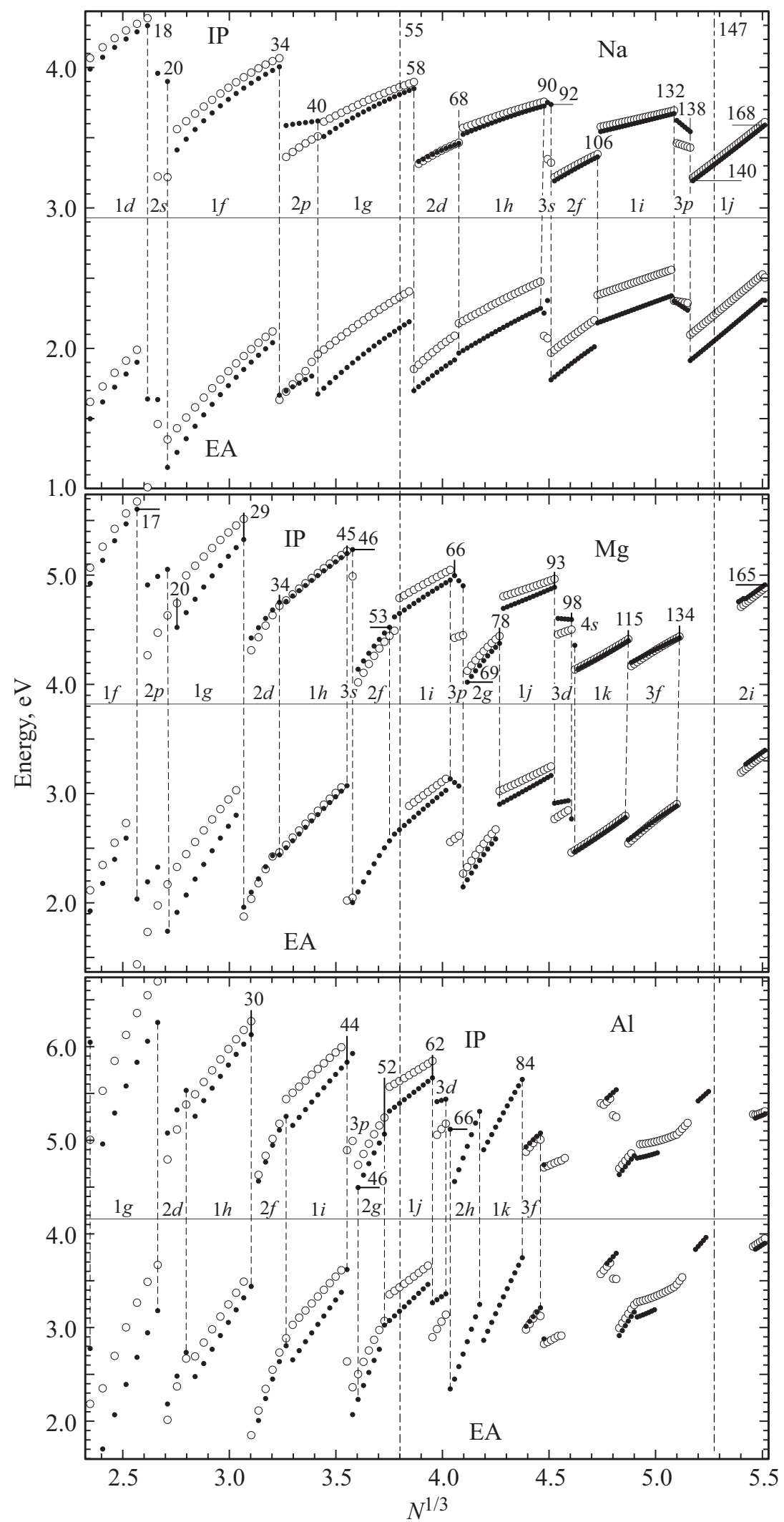

Рис. 2. Рассчитанные потенциалы ионизации IP и энергии прилипания ЕА для сплошных кластеров (сплошные кружки) и кластеров с моновакансией (пустые кружки). Указаны магические числа атомов, электронные оболочки, а также числа 55 и 147 , соответствующие 2-й и 3-й атомным оболочкам. 
Однако вследствие того, что концентрация равновесных внутренних вакансий в 3D-металлах экспоненциально зависит от обратной температуры, поверхностная энергия металла и работа выхода электронов должны содержать, помимо линейной температурной зависимости, обусловленной тепловым расширением, также экспоненциальную зависимость, которую легче наблюдать вблизи точки плавления 3D-металлов. Возможно, следует ожидать похожих температурных зависимостей и для характеристик кластеров. Способность различать эти зависимости для больших кластеров или островковых пленок определяется, в конечном счете, точностью эксперимента.

\section{Список литературы}

[1] Y. Kraftmakher. Phys. Rep. 299, 79 (1998).

[2] C. Freysoldt, B. Grabowski, T. Hickel, J. Neugebauer, G. Kresse, A. Janotti, C.G. Van de Walle. Rev. Mod. Phys. 86, 253 (2014).

[3] P.M. Agrawal, B.M. Rice, D.L. Thompson, J. Chem. Phys. 118, 9680 (2003).

[4] C. Hock, C. Bartels, S. Straßburg, M. Schmidt, H. Haberland, B. von Issendorff, A. Aguado. Phys. Rev. Lett. 102, 043401 (2009).

[5] V.V. Pogosov, E.V. Vasyutin. Nanotechnology 17, 3366 (2006).

[6] A.V. Babich, V.V. Pogosov. Surf. Sci. 604, 210 (2010).

[7] V.Z. Kresin, Y. Ovchinnikov. Phys. Rev. B 74, 024514 (2006).

[8] A. Halder, V.V. Kresin. Phys. Rev. B 92, 214506 (2015).

[9] P. Ziesche, J.P. Perdew, C. Fiolhais. Phys. Rev. B 49, 7919 (1994).

[10] В.В. Погосов. ФТТ 36, 2521 (1994).

[11] А.В. Бабич, П.В. Вакула, В.В. Погосов. ФТТ 56, 841 (2014).

[12] M. Sinder, D. Fuks, J. Pelleg. Phys. Rev. B 50, 2775 (1994).

[13] H. Delavari, H.R. Madaah Hosseini, A. Simchi. Physica B 406, 3777 (2011).

[14] C.C. Yang, S. Li. Phys. Rev. B 75, 165413 (2007).

[15] S.C. Hendy. Nanotechnology 18, 175703 (2007).

[16] G. Guisbiers. Nanoscale Res. Lett. 5, 1132 (2010).

[17] M. Itoh, V. Kumar, Y. Kawazoe. Phys. Rev. B 73, 035425 (2006).

[18] J. Akola, M. Manninen, H. Häkkinen, U. Landman, X. Li, L.-S. Wang. Phys. Rev. B 62, 13216 (2000).

[19] S. Chacko, D.G. Kanhere, S.A. Blundell. Phys. Rev. B 71, 155407 (2005).

[20] A.K. Starace, C.M. Neal, B. Cao, M.F. Jarrold, A. Aguado, J.M. López. J. Chem. Phys. 131, 044307 (2009). 